\title{
Erratum: U-geometry: SL(5)
}

\section{Jeong-Hyuck Park and Yoonji Suh}

Department of Physics, Sogang University,

Mapo-gu, Seoul 121-742, Korea

E-mail: park@sogang.ac.kr, yjsuh@sogang.ac.kr

ERRATUM TO: JHEP04(2013)147

ARXIV EPRINT: 1302.1652

The erratum essentially amounts to the multiplication of all the Ricci scalar expressions in the initial paper by factor two,

$$
R_{g} \quad \rightarrow \quad 2 R_{g}
$$

Consequently we have the following changes.

1. Eqs. (1.9) and (5.8) should be replaced by

$$
\mathcal{R}=e^{-\phi}\left[R_{g}-\frac{7}{2} \partial_{\mu} \phi \partial^{\mu} \phi+3 \square \phi+\frac{1}{2} e^{-\phi}\left(\nabla_{\mu} v^{\mu}\right)^{2}\right] .
$$

2. Eqs. (1.10) and (5.9) should be replaced by

$$
\int_{\sum_{4}} M^{-1} \mathcal{R}=\int \mathrm{d}^{4} x e^{-2 \phi} \sqrt{-g}\left(R_{g}+\frac{5}{2} \partial_{\mu} \phi \partial^{\mu} \phi-\frac{1}{48} e^{-\phi} F_{\kappa \lambda \mu \nu} F^{\kappa \lambda \mu \nu}\right) .
$$

3. In eqs. (1.8) and (5.2), the expression of $C_{\lambda \mu \nu}$ should be replaced by

$$
C_{\lambda \mu \nu}=\frac{1}{\sqrt{-g}} \epsilon_{\lambda \mu \nu \rho} v^{\rho}
$$

4. In eq. (5.2) the expression of $v^{\kappa}$ should be replaced by

$$
v^{\kappa}=\frac{1}{3 ! \sqrt{-g}} \epsilon^{\kappa \lambda \mu \nu} C_{\lambda \mu \nu}
$$

5. Footnote 8 should be ignored except "In our convention, $\epsilon^{0123}=1 . "$

All the above changes have been made in the latest arXiv version of the paper, arXiv:1302.1652. 